\title{
Karzinomtherapie mit dem Endoskop
}

In der Diagnostik und Therapie der Verdauungskrankheiten spielt die Endoskopie heute eine dominierende Rolle. Während die endoskopische Therapie in den vergangenen drei Jahrzehnten zahlreiche klassische chirurgische Verfahren ersetzt hat, erfährt die Diagnostik durch die Endoskopie derzeit neue Impulse, insbesondere im Bereich der Krebsfrüherkennung. Krebsdiagnostik impliziert Detektion, Staging und Grading. Für die Detektion bösartiger Schleimhautveränderungen ist die Endoskopie die Methode der Wahl. Biopsie und Histologie waren zur Bestätigung endoskopischer Befunde bislang unverzichtbar. Staging erfolgt zunehmend mit dem endoskopischen Ultraschall (EUS), während Grading nach wie vor die Domäne der Pathologie bleibt. Neueste Technologien, wie Narrow Band Imaging (NBI), Fluoreszenzendoskopie, Confocal Laser Scanning Microscopy (CLSM) und Zytoskopie ermöglichen Auffindung und Identifizierung dysplastischer Zellen, so dass eine virtuelle Histologie bzw. Zytologie in naher Zukunft schon während der endoskopischen Untersuchung erhalten werden kann. Die Größe des endoskopisch diagnostizierbaren Schleimhautkrebses wird dann nur 1-2 mm betragen, so dass die Entfernung mit der Biopsiezange sofort erfolgen kann. Zukunftsträchtig scheint die NBI-Filtertechnik zu sein, da damit eine noch bessere Darstellung der Schleim- hautoberfläche ohne Anfärbung möglich ist. Suspekte Schleimhautareale erscheinen dunkel und lassen sich dadurch leicht erkennen und direkt biopsieren. Die Untersuchungstechnik mit Hilfe des gebündelten Blaulichtes basiert auf der Darstellung der feinen oberflächlichen Kapillaren (Neoangiogenese), die sich bei Krebsgeschwülsten vermehrt bilden. Da aber der Krebs in einem sehr frühen Stadium noch nicht symptomatisch ist, lässt sich eine Früherkennung nur über das Screening bei Krebsrisikopersonen sinnvoll realisieren.

Nib Soehendra, Direktor der Klinik und Poliklinik für Interdisziplinäre Endoskopie, Universitätsklinikum Hamburg-Eppendorf
Weltweit führender Fortbildungskongress in Hamburg erreichte Teilnehmerrekord

\section{Der ENDO CLUB NORD 2006}

Zum 14. Mal trafen sich beim ENDO CLUB NORD, dem weltweit größten Kongress für LiveEndoskopie, internationale Experten aus 20 Ländern. Rund 2,700 Teilnehmer kamen ins Congress Center Hamburg (CCH), um sich von den Endoskopie-Teams der Asklepios Kliniken Altona und Barmbek und des UK Eppendorf auf den neuesten Stand der Entwicklungen zu bringen. Der weltweit größste Kongress für Live-Endoskopie stand ganz im Zeichen von technischen Innovationen, kontroversen Diskussionen und praktischen Anwendungen rund um die Endoskopie. Der ENDO CLUB NORD wird im nächsten Jahr in Hamburg fortgesetzt. Veranstatungstermin ist vom 9. bis 10. November 2007 im Congress Center Hamburg.

Weitere Informationen: www.endoclubnord.de

\section{Die Partner des ENDO CLUB NORD}

Das Pharmaunternehmen ALTANA Pharma AG und das Unternehmen OLYMPUS sind Partner des ENDO CLUB NORD Kongresses und unterstützen durch ihr Engagement die Entwicklungen auf dem Gebiet der Endoskopie. ALTANA Pharma ist eine international erfolgreiche Unternehmensgruppe, die auf Arzneimittel aus eigener Forschung setzt und sich dabei auf die Behandlung bedeutender Volkskrankheiten konzentriert. Im Bereich der Magen-Darm-Erkrankungen verfügt ALTANA Pharma mit dem Protonenpumpenhemmer Pantoprazol (Pantozol $\left.{ }^{\circledR}\right)$ über ein innovatives Therapeutikum, das derzeit das erfolgreichste Medikament aus deutscher Pharmaforschung ist. Pantoprazol ist in über 90 Ländern zugelassen. Über die eigene Forschung hinaus unterhält ALTANA Pharma zahlreiche Forschungskooperationen und engagiert sich seit Jahrzehnten in Förderprojekten für Wissenschaft und Bildung.

Olympus stellt einen Großteil aller weltweit klinisch eingesetzten Endoskope und Endoskopiesysteme her. Als erster Anbieter im Bereich der Medizintechnik ist das Unternehmen der Systemanbieter für starre, flexible und Endotherapie-Instrumente. Neben der langjährigen Unterstützung des ENDO CLUB NORD bietet Olympus darüber hinaus spezielle Seminare für Fach- und Pflegepersonal an (Olympus Akademie).

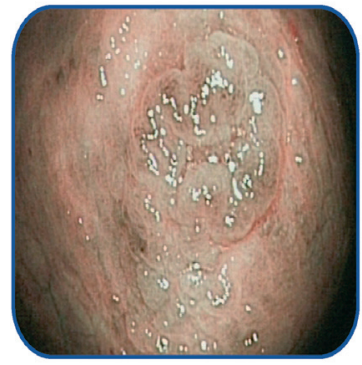

a

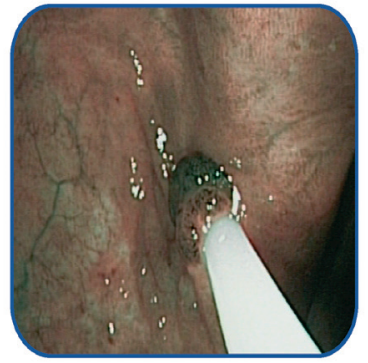

b

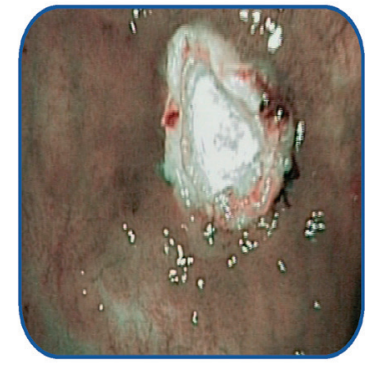

C
Abb. 1. a. Colitis ulcerosa in vollständiger Remission nach langjährigem Krankheitsverlauf. Mit Hilfe der NBITechnik konnte ein ca. 5 mm großes Schleimhautareal mit geringgradiger Displasie entdeckt werden. b. Endoskopische Abtragung. c. Zustand nach vollständiger Abtragung.
Impressum

Karzinomtherapie mit dem Endoskop Innovationen in Chirurgische Gastroenterologie INTERDISZIPLINÄR 22 | 4 | 06

(c) 2006 by S. Karger Verlag für Medizin und Naturwissenschaften $\mathrm{GmbH}$ Lörracher Straße 16a 79115 Freiburg, Deutschland

\section{Mit freundlicher Unterstützung durch} ALTANA Pharma AG und OLYMPUS GmbH.

Der Verlag und die Herausgeber der Zeitschrift übernehmen keine Verantwortung für diese Rubrik. Die Wiedergabe von Gebrauchsnamen, Handelsnamen, Warenbezeichnungen usw. in dieser Zeitschrift berechtigt auch ohne besondere Kennzeichnung nicht zur Annahme, dass solche Namen im Sinne der Warenzeichen- und Markenschutz-Gesetzgebung als frei zu betrachten wären und daher von jedermann benutzt werden dürfen. 